\title{
The Power Plant's Comprehensive Evaluation of Energy Saving Based on Entropy and ELECTRE Method
}

\author{
Pengfei Wang ${ }^{1, a}$, Changliang Liureb \\ ${ }^{1}$ School of Control and Computer Engineering, North China Electric Power \\ University,Baoding, 071000,China \\ ${ }^{2}$ State Key Laboratory of Alternate Electrical Power System with Renewable Energy Sources, \\ North China Electric Power University, Beijing,102206, China

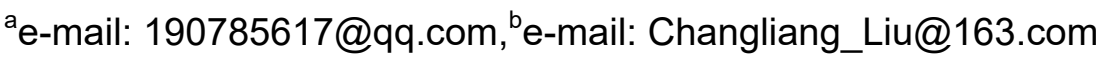

\section{Keywords: Thermal Plant Energy Conservation; Comprehensive Evaluation; Entropy; ELECTRE}

\begin{abstract}
With the national emphasis on energy conservation and environmental protection and as the primary energy consumption and emissions, more and more people pay attention on the energy saving of thermal power plant. In this paper, some relevant indicators for the energy saving of thermal power plant are selected ,and the improved ELECTRE based on entropy weight is applied to the comprehensive evaluation of energy saving and emission reduction in thermal power plant. Based on the information entropy, the weight coefficient of each index is determined, and the scientific and objectivity of the energy saving evaluation of thermal power plant are raised. Using ELECTRE to sort, the principle is simple and the effect is good Take five plants in an area as example, nine indicators are selected to build energy-related evaluation system. Analysis and calculation based on the evaluation model established in this paper, ultimate proof that the ELECTRE Method Based on Entropy in Comprehensive Evaluation of Power Plant in the energy saving is effective.
\end{abstract}

\section{Introduction}

In recent years, with the increasing of air pollution and the depletion of resources, the country have more attention on energy saving and environmental protection. As a major coal consumption and pollutant emissions, the energy saving and emission reduction of thermal power plant has been paid more and more attention by people. But at present, compared with the optimal operation of the thermal power plant, the comprehensive evaluation of energy saving and emission reduction in thermal power plants appears relatively backward. The paper [1] established the evaluation index system of energy saving and emission reduction in power industry, but did not give a specific evaluation method. The mathematical model of the economic comprehensive evaluation index of thermal power plant was constructed by paper [2] and paper [3]., but the single power plant can not reflect the level of energy utilization. In the paper [4], the theory of fuzzy set theory is used to evaluate the energy conservation status of thermal power plant, but this method is too dependent on the experience of experts and the degree of cognition and have poor objectivity. In the paper [5], the entropy weight fuzzy matter element evaluation model was established to evaluate the energy saving and emission reduction in thermal power plant. In the paper [6], the grey correlation projection method based on TOPSIS is applied to the comprehensive evaluation of energy saving and emission reduction in thermal power plant. However, this method is more complex and the application of TOPSIS method has its limitations. That is, when the evaluation object in the environment and its own conditions is changed, the index value is changed accordingly, which is likely to cause the change of the ideal point, so that the order of discharge also changes [7]. In this paper, the ELECTRE method based on entropy weight is applied to the comprehensive evaluation of energy saving and emission reduction in thermal power plant. The weight is determined by entropy weight method, which makes the evaluation more objective removals of subjective influence. A kind of super order relationship is established through the ELECTRE method, and then to sort the program. And the principle is simple, the effect is good and there is no limit to the application. In this paper, the 
ELECTRE method based on entropy weight provides a simple, accurate and reliable decision-making method for the comprehensive evaluation of energy saving and emission reduction in power plant. Finally, the effectiveness of the proposed method is proved by evaluating the energy saving and emission reduction of five power plants in a certain area.

\section{ELECTRE evaluation method based on entropy weight}

\section{Entropy Weight Method}

For the decision of multi attribute scheme, the determination of weight is a very important step in the process of evaluation. The weight reflects the importance of each index, and the weight of index is directly related to the overall contribution of this index and has a great influence on the final ranking results. The weight is reasonable or not plays a vital role on the rationality of evaluation results. If the weight of an index changed, It will affect the whole evaluation results. Therefore, the weight of the solution is a very important step in multi attribute decision making.

In this paper, we use the objective weighting method: entropy weight method. The entropy weight method is a method which to determine the index weights according to the amount of information provided by the observation value of each index [8]. In general, the entropy of an index is smaller indicating that the variation degree of the index value is bigger, the amount of information provided is more and the role in the comprehensive evaluation is greater, then the weight of the index should be bigger. The entropy method is a kind of objective weighting method and has simple calculation steps, and the effective use of the index data exclude the influence of subjective factors 。

Assuming that a system $\mathrm{X}$ may appear in several different states $\mathrm{x} 1, \mathrm{x} 2, \ldots, \mathrm{xm}, \mathrm{p}(\mathrm{xi})$ represents the probability of the emergence of the state $\mathrm{xi}(\mathrm{i}=1,2, \ldots, \mathrm{m})$, the information entropy $\mathrm{E}(\mathrm{X})$ of the system can be defined as:

$$
E(X)=-\frac{\sum p\left(x_{i}\right) \ln \left(p\left(x_{i}\right)\right)}{\ln m}
$$

In formula (1), $0 \leq \mathrm{p}\left(x_{i}\right) \leq 1$ and $\sum_{1}^{\mathrm{m}} \mathrm{p}\left(x_{i}\right)=1$; In particular, when $\mathrm{P}_{\mathrm{i}}=0, \mathrm{P}_{\mathrm{i}} \ln \mathrm{P}_{\mathrm{i}}=0$. The evaluation matrix composed of $\mathrm{N}$ evaluation scheme $\mathrm{m}$ index is $Y=\left(y_{i j}\right)_{m \bullet n}, \mathrm{i}=1,2, \ldots, \mathrm{m} ; \mathrm{j}=1,2, \ldots, \mathrm{n}$. The general procedure of determining weights by entropy weight method is:

(1)Calculate the proportion of each scheme under the $\mathrm{j}$ attribute, and get the normalized decision matrix.

$$
A=\left(\mathrm{a}_{i j}\right)_{m \times n}
$$

And the normalized matrix is obtained by the $0-1$ transform method, If the index $\mathrm{J}$ is a positive indicator, then

$$
\mathrm{a}_{i j}=\frac{y_{i j}-\min \left(y_{j}\right)}{\max \left(y_{j}\right)-\min \left(y_{j}\right)}
$$

If the index $\mathrm{J}$ is the inverse index, then

$$
\mathrm{a}_{i j}=\frac{\max \left(y_{j}\right)-y_{i j}}{\max \left(y_{j}\right)-\min \left(y_{j}\right)}
$$

(2)According to the definition of information entropy, the information entropy of the evaluation index of $\mathrm{J}$ is calculated. As shown in formula 5.

$$
E_{\mathrm{j}}=-\frac{\sum a_{i j} \ln \left(a_{i j}\right)}{\ln m}
$$

(3)Calculate the deviation degree: $\mathrm{d}$

$\mathrm{d}_{j}=1-E_{j}$

(4)Calculate the weight of each evaluation index. 


$$
\mathrm{w}_{j}=d_{j} / \sum_{i=1}^{n} d_{j}
$$

Therefore, the objective weight of each evaluation index is $w=\left\{w_{j}\right\},(j=1,2, \ldots, n)$.

\section{ELECTRE Method}

ELECTRE method is proposed by Roy [9] and then gradually improved and it is an effective method for multi attribute decision making. The key of ELECTRE method is to use the concept of super order relation, namely when the program A1 and A2 have no obvious advantages and disadvantages, decision makers believe that A1 is better than A2 to a certain degree of risk. In this way, we can get rid of the inferior scheme in the sense of super order by evaluating a series of super order relation [10]. There are many ways to establish outranking relationship. Here we use the method of net advantage value and net inferior position value proposed by Delft Van and Nijkamp and combined correction model of ELECTRE method proposed by S. M. Sun of Taiwan [11] and a simplified method based on ELECTRE ranking proposed by C. Q. Chen [12]. The steps are:

1)Identify strengths and weaknesses, $C_{\mathrm{kl}}=\left\{j \mid a_{k j} \geq a_{l j}\right\}, D_{k l}=\left\{j \mid a_{k j}<a_{l j}\right\}, C_{\mathrm{kl}}$ is label collection that the program $\mathrm{k}$ is better than the program 1 , and $D_{\mathrm{kl}}$ is label set that the program $\mathrm{k}$ is inferior to the program 1 .

(2)Seek the relative superiority index and the relative inferiority index. The $c_{k l}, d_{k l}$ have the following definition.

$$
\mathrm{c}_{k l}=\sum_{j \in C_{k l}} w_{j} / \sum_{j=1}^{n} w_{j}
$$

(8)

$\sum_{j=1}^{n} w_{j}=1, \quad c_{k l}$ is called the relative dominance index of the scheme 1 to the scheme $k$.

$$
\mathrm{d}_{k l}=\frac{\max _{j \in D_{k l}}\left|w_{j}\left(a_{k j}-a_{l j}\right)\right|}{\max _{j \in J}\left|w_{j}\left(a_{k j}-a_{l j}\right)\right|}
$$

(9)

$\mathrm{d}_{k l}$ is called the relative inferiority index of the scheme $\mathrm{k}$ to the scheme $1 . \mathrm{c}_{k l}$ contains only the index weight information, and $\mathrm{d}_{k l}$ not only contains the weight information, but also has the index value information. Thus the relative dominance index and the relative disadvantage index are not complementary.

(3) The correction of the disadvantage of the matrix.

Based on the discussion of the Taiwan scholar S. M. Sun, the inferior matrix is redefined.

$$
\mathrm{d}_{k l}=1-d_{k l}
$$

(4)Modified weighted sum matrix.

Due to disadvantage matrix of traditional ELECTRE method is modified, the elements in the modified disadvantage matrix are the same as the elements in the dominant matrix, the greater the value, the higher the degree of preference. Therefore, we can get the modified weighted sum matrix through multiplication of elements in the corresponding position of the dominant matrix and the modified matrix.. the formula is:

$$
\mathrm{e}_{k l}=c_{k l} d_{k l}
$$

(5)The value of net advantage

According to the concept of net advantage value proposed by Delft Van and Nijkamp, the method is as follows: 


$$
\mathrm{c}_{K}=\sum_{\substack{l=1 \\ l \neq k}}^{m} e_{k l}-\sum_{\substack{l=1 \\ l \neq k}}^{m} e_{l k}
$$

$\mathrm{c}_{K}$ is subtraction between the weighted advantage of the scheme $\mathrm{k}$ relative to the other schemes and the weighted advantage of other schemes relative to the scheme $\mathrm{k}$, and it reflects the weighted total net advantage of the scheme. The greater the $\mathrm{k}$, the solution will be more optimal.

(6)Sort.

We can sort according to the weighted total net advantage value, then we can get the final order of the enterprise from the superior to the inferior.

\section{Comprehensive Evaluation of Energy Saving and Emission Reduction}

\section{Establishment of Evaluation System}

According to the actual operation of the thermal power plant, there are the following principles in the selection of indicators: 1) indicators comprehensive and not repeated; 2) it is both representative and operational, and easy to collect;Through a lot of reading related literature, we choose the following 9 indicators to build the evaluation index system: Standard Coal Consumption Rate, Power Consumption Rate of Plant, Comprehensive Energy Consumption of Ten Thousand RMB Output Value, Repeated Utilization Ratio of Industrial Water, The New Water Consumption Rate of Per $\mathrm{kWh}$, The Discharge Rate of Wastewater of Per $\mathrm{kWh}, \mathrm{SO}_{2}$ Emissions Rate of per $\mathrm{kWh}$, $\mathrm{NO}_{\mathrm{X}}$ Emissions Rate of Per kWh, Dust Emissions Rate of Per kWh. Among them, the recycling rate of industrial water is a positive indicator, the greater the value, the better, the remaining 8 indicators are negative indicators, the greater the value, the fire power plant energy saving effect is not good. These 9 indicators reflect the energy-saving and emission reduction effect in all aspects of the thermal power plant. Comprehensive consideration, these 9 indicators can fully reflect the level of energy efficiency of power plants.

\section{Evaluation Process}

Using ELECTRE method based on entropy weight to evaluate the effect of energy saving and emission reduction in thermal power plant, the general steps are:

(1)Determine the evaluation index, and list the data list of the matrix $\mathrm{X}$ to be evaluated.

(2)Application formula (3) and formula (4) to normalize the matrix X

(3)Application type (5) - (7) to get the weight of each index W.

(4)According to the formula (8) - (12) to obtain the net advantage value of each evaluation scheme.

(5)According to the value of the net advantage of each program to sort.

\section{Evaluation Results}

In this paper, the ELECTRE method based on entropy weight is used to evaluate the energy saving and emission reduction effect of 5 thermal plants in a certain area. The energy saving and emission reduction targets and operation data of 5 thermal plants are shown in Table 1. The process of comprehensive evaluation will be realized by MATLAB programming.

According to the characteristic of index (positive and negative index), the standard 0-1 transform method that mentioned above is used to standardize the data in Table 1, and the decision matrix of standardized are shown in Table 2.

According to the above information entropy method to obtain the objective weight and through the MATLAB, we get the objective weight of each attribute $\mathrm{W}=\left(\begin{array}{l}0.09640 .08880 .09410 .13800 .1340 \\ 0.080\end{array}\right.$ 0.13470 .10370 .12560 .0847 ), the order of weights and the order of indicators are in agreement。

According to the ELECTRE sorting method to prepare the MATLAB program, the modified weighted sum matrix is obtained according to formula (11). 
Table 1 The evaluation indexes of energy saving and emission reduction data of five power plant

\begin{tabular}{|c|c|c|c|c|c|c|c|c|c|}
\hline \multicolumn{5}{|c|}{ Indicator } & One & Two & Three & Four & Five \\
\hline \multicolumn{5}{|c|}{ Standard Coal Consumption Rate } & 396.00 & 323.14 & 327.45 & 289.08 & 318.45 \\
\hline \multicolumn{5}{|c|}{ Power Consumption Rate of Plant } & 7.43 & 7.87 & 7.59 & 6.80 & 6.6 \\
\hline \multicolumn{5}{|c|}{$\begin{array}{c}\text { Comprehensive Energy Consumption of Ten } \\
\text { Thousand RMB Output Value }\end{array}$} & 9.806 & 7.720 & 10.310 & 4.405 & 9.731 \\
\hline \multicolumn{5}{|c|}{ Repeated Utilization Ratio of Industrial Water } & 96.35 & 98.34 & 97.60 & 91 & 97.60 \\
\hline \multicolumn{5}{|c|}{ Consumption Rate of New Water } & 2.44 & 2.3 & 2.65 & 3.29 & 3.3 \\
\hline \multicolumn{5}{|c|}{ Discharge Rate of Wastewater } & 1.5 & 0.014 & 0.073 & 0.2 & 1.88 \\
\hline \multicolumn{5}{|c|}{ Emission Rate of SO2 } & 13.2 & 9.9 & 4.1 & 5.2 & 12.3 \\
\hline \multicolumn{5}{|c|}{ Emission Rate of NOX } & 8 & 1.75 & 1.20 & 5.7 & 1.8 \\
\hline \multicolumn{5}{|c|}{ Emission Rate of Dust } & 0.4 & 0.185 & 0.459 & 0.7 & 0.656 \\
\hline \multicolumn{10}{|c|}{ Table 2 Standardized decision matrix } \\
\hline \multicolumn{5}{|c|}{ Indicator } & One & Two & Three & Four & Five \\
\hline \multicolumn{5}{|c|}{ Standard Coal Consumption Rate } & 0 & 0.6814 & 0.6411 & 1 & 0.7253 \\
\hline \multirow{2}{*}{\multicolumn{5}{|c|}{$\begin{array}{c}\text { Power Consumption Rate of Plant } \\
\text { Comprehensive Energy Consumption of Ten } \\
\text { Thousand RMB Output Value }\end{array}$}} & 0.3465 & 0 & 0.2205 & 0.8425 & 1 \\
\hline & & & & & 0.0854 & 0.4386 & 0 & 1 & 0.0981 \\
\hline \multicolumn{5}{|c|}{ Repeated Utilization Ratio of Industrial Water } & 0.7289 & 1 & 0.8992 & 0 & 0.8992 \\
\hline \multicolumn{5}{|c|}{ Consumption Rate of New Water } & 0.8600 & 1 & 0.6500 & 0.01 & 0 \\
\hline \multicolumn{5}{|c|}{ Discharge Rate of Wastewater } & 0.2036 & 1 & 0.9684 & 0.9003 & 0 \\
\hline \multicolumn{5}{|c|}{ Emission Rate of SO2 } & 0 & 0.3587 & 1 & 0.8696 & 0.0978 \\
\hline \multicolumn{5}{|c|}{ Emission Rate of NOX } & 0 & 0.9191 & 1 & 0.3382 & 0.9118 \\
\hline \multicolumn{5}{|c|}{ Emission Rate of Dust } & 0.5825 & 1 & 0.4680 & 0 & 0.0854 \\
\hline \multirow{5}{*}{\multicolumn{2}{|c|}{$\left.\begin{array}{llll}e_{31} & e_{32} & e_{34} & e_{35} \\
e_{41} & e_{42} & e_{43} & e_{45} \\
e_{51} & e_{52} & e_{53} & e_{54}\end{array}\right)$}} & 0 & 0 & 0.0547 & $0.0023)$ & & & & \multirow{5}{*}{$(13)$} \\
\hline & & 0.6683 & 0 & 0.2824 & 0.2777 & & & & \\
\hline & & 0.4643 & 0.0937 & 0.1740 & 0.3383 & & & & \\
\hline & & 0 & 0 & 0 & 0 & & & & \\
\hline & & 0 & 0 & 0 & 0.0098 & & & & \\
\hline
\end{tabular}

According to the formula (12) and (13), we can get the net advantage shown in Table 3.

Table 3 The net Advantage Value of power plant

\begin{tabular}{|c|c|c|c|c|c|}
\hline Net advantage value & One plant & Two plant & Three plant & Four plant & Five plant \\
\hline C & -1.0756 & 1.1347 & 1.0703 & -0.5209 & -0.6085 \\
\hline
\end{tabular}

From table 3: The weighted total net advantage of the two thermal plant is the largest, so the energy saving and emission reduction effect of the two thermal plant is the best. According to the size of the weighted total net value of the thermal plant to be evaluated, we can get the order that five thermal plant energy saving and emission reduction effect from the first to the inferior: \#2 thermal plant $>\# 3$ thermal plant $>\# 4$ thermal plant $>\# 5$ thermal plant $>\# 1$ thermal plant..

Combined with the operation data of thermal plant, \#2thermal plant is worse than \#1 thermal plan only in terms of thermal plant electricity rate, and \#2 thermal plants are better than \#1 thermal plant in other 8 indicators. Therefore, the energy-saving emission reduction effect of $\# 2$ thermal plant is better than \#1 thermal plant.. Although the \#5 thermal plant is better than \#3 thermal plant in standard coal consumption rate, thermal plant power consumption rate and comprehensive energy consumption of ten thousand RMB output value. but in the other five areas, \#5 thermal plant is inferior to \#3 thermal plant. Thus reducing the overall results, \#5 thermal plant arranged after the \#3 thermal plant. In fact, \#5 thermal power plant unit is relatively old and the power load is small, and \#2 thermal power plant is a new power plant, energy saving measures are better.

\section{Concluding Remarks}

The comprehensive evaluation of energy saving and emission reduction in thermal power plant is a complicated problem. In this paper, by selecting 9 representative indexes of energy saving and emission reduction in thermal power plant and the effect of energy saving and emission reduction of 
5 power plants is evaluated by entropy weight ELECTRE method. The weight is determined by entropy weight method, which overcomes the influence of subjective factors on the evaluate results, so that the evaluation results are more objective. And the use of ELECTRE method to sort the energy saving and emission reduction effect of power plant, and this method is simple, easy to program, easy to be operated by the operator to master. It is proved that the method can comprehensively evaluate the effect of energy saving and emission reduction, and this method provides an effective method for the comprehensive evaluation of energy saving and emission reduction in thermal power plant.

\section{References}

[1] Y. W. Gu, C. H. Li, The Research on Evaluation Index System of Energy Saving and Emission Reduction in Electric Power Industry, Journal of Shenyang University of Technology(The Social Science Edition) ,2013,6(1):73-76.

[2] S. Z. Li, Y. M. Wang, L. H. Ma, Economic evaluation of coal fired power plant based on technology, economy and environment, Thermal Power Generation, 2009,38(5):1-4.

[3] Y. M. Wang, L. P. Li, W. J. Pan, Study on Comprehensive Evaluation Index of Operation Economy of Coal Fired Power Plant, Changsha Journal of Science \& Technology (NATURAL SCIENCE EDITION), 2006,3(1):47-51.

[4] Y. Y. Man, C. G. Fu, State Evaluation of Thermal Power Plant Based on Fuzzy Comprehensive Evaluation, China Electrical Engineering, 2009,29(5):5-10.

[5] L. H. Cao, W. T. Cui, J. J. Xu, Application of Entropy Weight Fuzzy Matter Element Model to The Comprehensive Evaluation of Energy Saving and Emission Reduction in Thermal Power Plant, 2015, 44(1):54-63.

[6] J. L. Yuan, J. H. Zhang, T. H. Wang, Energy saving comprehensive evaluation and weight sensitivity analysis of thermal power plant based on grey correlation projection method based on TOPSIS, 2015,35(5):404=411.

[7] J. J. Li, Multi objective evaluation method for sustainable development of thermal power enterprises, Business School of Hunan University, Changsha, China, 2006.

[8] Z. W. Wang, K. Tang, L. Yan, Comprehensive decision making of thermal power plant site based on entropy weight TOPSIS method, Safety and Environmental Engineering, 2011, 18(5):103-106.

[9] Roy. B. How outranking relation help multiple criteria decision making[C]// J. L. Cochrane, M. Zeleny. Multiple Criteria Decision Making .South Carolina: University of South Carolina Press, 1973:179-201

[9] J. Zuo, Multiple Objective Decision Analysis, Hangzhou, China, Zhejiang University Press,1991.

S. M. Sun, The Study of ELECTRE under Fuzzy Environment, Institute of Industrial Engineering of Southeast University, Taiwan, China, 2000.

[10] Y. Gao. C. Q. Chen, A simplified method based on ELECTRE ranking, Statistical Decision, 2006, (10): 37-39.. 